\section{Etiology of Bipolar Disorder Across the Lifespan: Essential Interplay With Diagnosis, Classification, and Assessment}

Benjamin L. Hankin, University of Denver

Bipolar disorder has garnered increasing attention as many argue that rates of bipolar disorder are skyrocketing and the definition of the classic bipolar disorder phenotype should be expanded, especially among children and adolescents. Understanding the psychosocial etiologies of bipolar disorder across the lifespan is critically important, and Alloy and colleagues' (2009) scholarly review makes an important contribution. Given the debate and controversy surrounding the description, diagnosis, and phenotype of bipolar disorder, having an accurate, reliable, and valid classification for definition, diagnosis, and assessment is critical for explicating potential etiology. Likewise, advanced understanding of etiology, especially when grounded in basic psychological science as Alloy and colleagues' review is, can importantly inform clinical phenomenology, course, assessment, and intervention. In summary, there is an essential interplay among description, classification, assessment, etiology, and intervention, such that a deeper understanding of all these areas is necessary for

Address correspondence to Benjamin L. Hankin, Department of Psychology, University of Denver, 2155 South Race St., Denver, CO 80208. E-mail: ben.hankin@psy.du.edu. advancing an empirically based practice of assessment and intervention.

Key words: bipolar disorder, etiology, diagnosis, classification, assessment. [Clin Psychol Sci Prac 16: $227-$ 230, 2009]

$M$ any scientists, clinicians, and laypeople alike are arguing that prevalence and incidence rates of bipolar disorder (BD) are skyrocketing. The media and popular press are increasingly publishing books and articles as well as releasing news stories about the astronomical rise in BD, especially among children and adolescents. Once considered primarily an adult-onset disorder, the rate in which pediatric BD has been diagnosed and treated in youth has more than doubled in the last 10 years (Youngstrom, Youngstrom, \& Starr, 2005). More than 100,000 children were being medicated for BD in 2001 (Hellander, 2002). Since Biederman and colleagues' (e.g., Biederman et al., 1995; Wozniak et al., 1995) research suggesting that pediatric BD may be underdiagnosed, and others proposing that the symptoms required for a diagnosis of pediatric BD may need to be expanded to account for potential developmental differences in core symptom expression and manifestation between youth and adults, the amount of research published on pediatric BD has expanded rapidly (Carlson \& Meyer, 2006; Duffy, 2007; Luby \& Belden, 2006; Youngstrom, Birmaher, \& Findling, 2008; Youngstrom, Meyers, Youngstrom, Calabrese, \& Findling, 2006). As these recent reviews make clear, classic BD clearly exists and has modal onset in adolescence or early adulthood, yet controversy and debate concerning core definition, description, and assessment of pediatric BD continue. Such uncertainties 
over basic and fundamental issues, especially clinical phenomenology, diagnosis, and assessment, have clear and important implications for understanding course, etiology, and intervention.

Capturing the growing public interest in $\mathrm{BD}$, especially pediatric $\mathrm{BD}$, a recent Public Broadcasting Station documentary, entitled The Medicated Child, explored this phenomenon, delineated various issues that accompany the increased attention and rising rates of $\mathrm{BD}$, and raised vital questions concerning basic issues for understanding $\mathrm{BD}$, especially its definition, diagnosis, classification, assessment, etiology, and treatment. For example, The Medicated Child documentary shows a for-profit business that provides "whole brain scans" for youth, demonstrably based on descriptive neuroimaging research, for the expressed purpose of early detection of $\mathrm{BD}$, before any actual symptoms are manifest, so that "at-risk" children can be prescribed mood stabilizers and antipsychotic medications to prevent potential onset of frank BD. Clearly, this practice of "whole brain scans" to identify at-risk youth with the goal of deflecting the possible BD trajectory via adult psychotropic medications raises serious questions: Does this practice of early detection go beyond the known scientific knowledge on diagnosis, assessment, causes, and treatment of $\mathrm{BD}$ ? Does this practice carry risks (e.g., likely side effects from adult antipsychotic medications and mood stabilizers) that outweigh possible benefits (e.g., probability of forestalling onset of BD)? Having a solid understanding of BD's etiology across the lifespan can elucidate such questions.

It is against this background with impassioned rhetoric, both in the public square and in scientific circles, on the description, definition, diagnosis, classification, assessment, cause, and treatment of BD that this special issue in Clinical Psychology: Science and Practice and the specific article on etiology of BD by Alloy, Abramson, Urosevic, Bender, and Wagner (2009) can be considered. Alloy and colleagues' scholarly review, grounded in basic psychological science with clear etiological and translational implications, shines needed light on the psychosocial factors and processes that may contribute to the onset, course, maintenance, and manifestation of bipolar spectrum disorders, predominantly in adults. Among the many laudable features of their outstanding review include the focus on longitudinal predictors of bipolar spectrum disorders to explicate pathways and processes, a firm theoretical framework rooted in basic psychological science, and an organizational structure along a distalproximal continuum.

These features are consistent with and promote a developmental psychopathology perspective for understanding the causes, course, expression, and outcomes for BD. Employing a developmental psychopathology framework is important given the various controversies surrounding pediatric BD (e.g., Carlson \& Meyer, 2006; Duffy, 2007; Youngstrom et al., 2006). This perspective is well positioned for illuminating and clarifying core debates swirling around the field of pediatric BD, including definition, symptom expression, diagnosis, classification, assessment, course and continuity into adolescence and adulthood, multiple causes and pathways (i.e., equifinality and multifinality), and intervention. As several of these topics (e.g., diagnosis, assessment, treatment) are addressed in other articles in this special issue, this commentary will not delve into them.

Still, it is important to highlight the crucial point that all of these topics, including accurate classification, diagnosis, description, and assessment, critically inform a developmental psychopathological understanding of the etiology of BD. In turn, a deeper knowledge of etiology, via an iterative bootstrapping process, can enhance comprehension of accurate and appropriate classification of $\mathrm{BD}$ across the lifespan. Clearly, having a reliable and valid diagnosis of $\mathrm{BD}$ is necessary for etiological research because inappropriate specification of the BP phenotype and inaccurate assessment of $\mathrm{BP}$ as the outcome will yield questionable and potentially faulty knowledge about etiological processes. On the other hand, having a theoretically based and empirically supported model of BD, as articulated well by Alloy and colleagues (2009), can importantly inform the assessment, course prediction, and treatment of $\mathrm{BD}$, as these features of $\mathrm{BD}$, combined with etiology, are part of the larger nomological network that is necessary for explicating the latent construct of any psychiatric disorder in a classification system (Follette \& Houts, 1996; Skinner, 1981), especially for BD, at deeper and multiple levels of analysis.

To establish validity for any psychiatric disorder, the classic criteria outlined by Robins and Guze (1970), including clinical phenomenology, etiology (e.g., genetics), course, and treatment response continue to be the field's 
signposts. The necessary interplay among description, diagnosis, assessment, course, etiology, and intervention is vital for advancing knowledge on the eventual goal of achieving a scientifically sound, reliable, and valid classification for BD. As the broad discipline of clinical psychology continues to embrace and adapt empirically based assessment (Youngstrom, Youngstrom, Findling, \& Calabrese, 2005) and intervention practices (e.g., Silverman \& Hinshaw, 2007) for BD and other psychopathologies, having an accurate, reliable, and valid classification system for describing the $\mathrm{BD}$ phenotype, recommending assessment practices, detailing etiological correlates and mechanisms, and prescribing efficacious interventions become of paramount importance. By understanding the etiology of $\mathrm{BD}$, the field can disconfirm potentially faulty ideas and practices. In this light, Alloy and colleagues' (2009) state-of-the-art review on prospective etiological predictors of the onset, course, and manifestation of BD spectrum disorders thus provides crucial information for scientists, clinicians, and the public. In summary, these different areas of research (description, assessment, etiology, and treatment) tie into each other and can create, in totality, a structure of knowledge that may further deepen the field's understanding of and advance efficacious treatments for BD.

Although Robins and Guze (1970) originally highlighted the predominant influence of genetics and family history as the essential etiological markers, it is clear that psychosocial factors and processes are equally important for providing a coherent and comprehensive understanding of the etiology of BD. As an enormous literature on the genetics of $\mathrm{BD}$ exists and continues to make important advances (e.g., Faraone, Glatt, \& Tsuang, 2003), it is equally important to complement etiological understanding of BD with psychosocial predictors. Indeed, the empirical literature reviewed by Alloy and colleagues (2009), with an emphasis on longitudinal predictors of BD based on prospective designs that control for initial symptom levels to predict change and course over time, is exceptionally impressive and provides valuably important data for predicting risk and course of BD. Clearly, genetic and neurobiological biomarker research is important for understanding etiology of BD at a different and complementary level of analysis, yet it is noteworthy that remarkably few genetic or neurobiological biomarker studies use rigorous longitudinal designs to predict onset and course of BD over time. As such, the corpus of knowledge on psychosocial longitudinal predictors and the use of prospective designs, especially when those involve multiple waves of follow-up data to carefully track developmental change and course over time, advances our understanding of etiology because prospective prediction addresses concerns about any particular psychosocial etiological risk factor being merely a correlate of $\mathrm{BD}$. Ideally, the research investigating genetic and neurobiological influences for BD would likewise employ longitudinal designs, especially multiwave follow-ups, to determine whether putative biomarkers function as cause, consequence, or concomitant of BD and would longitudinally examine how different genetic and neurobiological influences affect the developmental course and expression of BD over time.

In summary, given the intensifying national dialogue and marked controversies surrounding the possibly expanding nature and diagnosis of $\mathrm{BD}$, especially pediatric $\mathrm{BD}$, accompanied by increasing use of off-label prescriptions of adult psychotropic medications to treat pediatric and young-adult BD, it is essential that clinical scientists and practitioners have an empirically informed and theoretically grounded understanding of potential causes of the onset, course, and varied expression of bipolar spectrum disorders across the lifespan. Moreover, the interplay of having an accurate and valid etiological understanding of $\mathrm{BD}$, especially when based on rigorous longitudinal designs to track onset and course over time, combined with Robins and Guze's (1970) other criteria of descriptive clinical phenomenology, course, and treatment response, all interweave to lead toward an empirically informed structure of knowledge. Such comprehension can ultimately culminate in an accurate and appropriate classification system for BD at a latent level across the lifespan. A valid classification system communicates to scientists, clinicians, and the public alike what $\mathrm{BD}$ is, how to assess it, what its course is, what causes it, and how best to intervene. With their scholarly review of longitudinal predictors of BD, Alloy and colleagues (2009) have provided the field with invaluable information from which empirically informed decisions concerning assessment, diagnosis, and treatment can be made as accurately as possible given the current state of knowledge. Clearly, scientific, clinical, and public interest in BD will continue, and armed with the knowledge contained within 
this review, we are in a stronger position to make wiser and more informed decisions for classifying, diagnosing, assessing, predicting course, and treating $\mathrm{BD}$ across the lifespan.

\section{REFERENCES}

Alloy, L. B., Abramson, L. Y., Urosevic, S., Bender, R. E., \& Wagner, C. A. (2009). Longitudinal predictors of bipolar spectrum disorders: A behavioral approach system perspective. Clinical Psychology: Science and Practice, 16(2), 206-226.

Biederman, J., Wozniak, J., Kiely, K., Ablon, S., Faraone, S., Mick, E., et al. (1995). CBCL clinical scales discriminate prepubertal children with structured interview-derived diagnosis of mania from those with ADHD. Journal of the American Academy of Child and Adolescent Psychiatry, 34, 464-471.

Carlson, G., \& Meyer, S. E. (2006). Phenomenology and diagnosis of bipolar disorder in children, adolescents, and adults: Complexities and developmental issues. Development and Psychopathology, 18, 939-969.

Duffy, A. (2007). Does bipolar disorder exist in children? A selected review. Canadian Journal of Psychiatry, 52, 409-417.

Faraone, S. V., Glatt, S. J., \& Tsuang, M. T. (2003). The genetics of pediatric-onset BD. Biological Psychiatry, 53, 970-977.

Follete, W. C., \& Houts, A. C. (1996). Models of scientific progress and the role of theory in taxonomy development: A case study of the DSM. Journal of Consulting and Clinical Psychology, 64, 1120-1132.

Hellander, M. (2002). Lithium testing in children: A public health necessity. Washington, DC: Testimony to the Food and Drug Administration.

Luby, J. L., \& Belden, A. (2006). Defining and validating bipolar disorder in the preschool period. Development and Psychopathology, 18, 971-988.
Robins, E., \& Guze, S. B. (1970). Establishment of diagnostic validity in psychiatric illness: Its application to schizophrenia. American Journal of Psychiatry, 126, 983-987.

Silverman, W. K., \& Hinshaw, S. P. (2007). The second special issue on evidence-based psychosocial treatments for children and adolescents: A 10-year update. Journal of Clinical Child and Adolescent Psychology, 37, 1-7.

Skinner, H. A. (1981). Toward the integration of classification theory and methods. Journal of Abnormal Psychology, 90, 6887.

Wozniak, J., Biederman, J., Kiely, K., Ablon, J. S., Faraone, S. V., Mundy, E., et al. (1995). Mania-like symptoms suggestive of childhood-onset bipolar disorder in clinically referred children. Journal of the American Academy of Child and Adolescent Psychiatry, 34, 867-876.

Youngstrom, E., Meyers, O., Youngstrom, J. K., Calabrese, J. R., \& Findling, R. L. (2006). Diagnostic and measurement issues in the assessment of pediatric bipolar disorder: Implications for understanding mood disorder across the life cycle. Development and Psychopathology, 18, 9891021.

Youngstrom, E. A., Birmaher, B., \& Findling, R. L. (2008). Pediatric bipolar disorder: Validity, phenomenology, and recommendations for diagnosis. Bipolar Disorders, 10(Suppl. 1, Pt. 2), 194-214.

Youngstrom, E. A., Youngstrom, J. K., Findling, R. L., \& Calabrese, J. R. (2005). Evidence-based assessment of pediatric bipolar disorder. Journal of Clinical Child and Adolescent Psychology, 34, 433-448.

Youngstrom, E. A., Youngstrom, J. K., \& Starr, M. (2005). Bipolar diagnoses in community mental health: Achenbach CBCL profiles and patterns of comorbidity. Biological Psychiatry, 58, 569-575.

Received February 22, 2009; accepted February 23, 2009. 